\title{
Risk Factors for Tuberculosis Conversion in a State Prison
}

\author{
Robert Hung, B.S.* , Steven Shelton, M.D., Gary Rischitelli, M.D., M.P.H., J.D.
}

\begin{abstract}
A case-control study determined the risk factors for latent tuberculosis (TB) conversion among Oregon Department of Correction (ODOC) inmates from July 2000 - July 2001. The first objective was to identity the converters. These were inmates who tested negative for the Purified Protein Derivative (PPD) skin test on entry and subsequently tested positive on annual testing. The second objective was determining the risk factors for conversion by comparing the converters with randomly selected controls. The Correctional Information System (CIS) and Mental Health databases were accessed to obtain health and demographic information. With ninety-nine percent of PPD positive inmates on anti-tuberculosis medications, nearly all male inmates who tested positive from July 00-01 $(\mathrm{n}=307)$ were identified through the ODOC pharmacy records. A medical chart review (276 of 307 or $90 \%)$ separated the converters $(n=72)$ from the reactors who tested positive on entry $(n=123)$ and the prior positives on medications $(n=81)$. The conversion rate was 5.0 per 1,000 person-years. Differences between the cases (converters) and controls were analyzed using multivariate logistic regression. The converters were 6 times more likely to be Latino $(p<.005)$ vs. Caucasian, over 19 times less likely to live in medium vs. minimum $(\mathrm{p}<.001)$ or maximum vs. minimum $(\mathrm{p}<.001)$ security prisons, and over 5 times less likely to live in a medium vs. low $(.012<p<.031)$ or high vs. low $(.002<p<.007)$ density prison. They had 1.4-1.5 times fewer PPD skin tests $(.002<p<.009)$ and lived in 1.5-1.7 times fewer prisons $(.005<p<.017)$. Age, education, county of incarceration, number of incarcerations, and number of visitors were not found to be significant variables. The results revealed a low conversion rate compared to other U.S. prisons. Prison health officials should consider performing two-step skin testing in order to distinguish the booster phenomenon from intramural conversion.
\end{abstract}

\section{INTRODUCTION}

Worldwide, tuberculosis (TB) is the second leading cause of death from a single infectious agent (1). Onethird of the world population is infected with Mycobacterium tuberculosis and causes were multifactorial: decreased funding for tuberculosis surveillance, increased immigration from areas of high TB prevalence, the unfortunate rise in HIV/AIDS, and major outbreaks in congregate settings such as prisons. Prisons had three times the rate of pulmonary TB than

\footnotetext{
* To whom correspondence should be addressed: 255 SW Harrison Street, \#7G, Portland Oregon, U.S.; Email: hungr@ohsu.edu.
}

the general population and in some New York, New Jersey, and California prisons, the incarcerated were 6-11 times more likely to develop active TB than the non-incarcerated (3-5).

In response to the outbreaks, the Centers for Disease Control (CDC) developed guidelines for correctional facilities in 1989 and again in 1995 (6-7). In the first guideline, the increased risk for active TB due to coinfection with HIV was highlighted. In the second guideline, the same principles of surveillance, namely screening, containment, and assessment, were emphasized. The basic principles revolved around yearly PPD skin testing, treatment with prophylactic 
medications, containment of active cases, and periodic assessments through incidence studies such as this one. Based on these recommendations, the ODOC instituted yearly PPD skin testing in 1990 .

With increased surveillance of high-risk populations such as inmates, immigrants, minorities, and the immuno-suppressed, the incidence of active TB in the U.S. decreased from 9.8 cases per 100,000 in $1993(\mathrm{n}=$ $25,287)$ to 5.2 cases per 100,000 in $2002(\mathrm{n}=15,078)$. (8) In Oregon, the incidence was even lower, with a rate of 3.2 per 100,000 in $2002(n=111)(9)$. Only a few active cases were found in the Oregon Department of Corrections (ODOC). From 1995-2001, there was only one case in each of 1997, 1998, and 2001 (9).

The decrease in incidence ushered a new paradigm with regards to TB control. In May 2000, the Institute of Medicine (IOM) issued a report entitled, "Ending Neglect: The Elimination of Tuberculosis in the United States" (10). The report detailed the multi-factorial strategies necessary to prevent TB resurgence and decisively eradicate TB in the U.S.. Eradication was defined as $<0.1$ case per 100,000 person-years. The basic principles revolved around surveillance, applied research, prevention and control, and infrastructure. A shift from active to latent $\mathrm{TB}$ screening was emphasized. The focus was on preventing active TB by detecting and treating latent TB. By doing so, the reservoir of Mycobacterium tuberculosis could be essentially eliminated.

In the ODOC, health officials wished to know the extent of TB transmission and the risk factors for latent TB conversion. The known risk factors in other prisons and jails were the following: 1) exposure to an active case, 2) increased crowdedness, 3 ) increased duration of stay, 4) being housed in multiple institutions, and 5) being incarcerated multiple times (11-13). With this information, they could increase the frequency of skin testing in the high-risk inmates. In addition, the baseline conversion rate could be established and the possible reasons for conversion explored. Of note, the booster phenomenon has been known to cause initial false negative tests. A 'booster' is a person with TB whose immune system is unable to elicit a positive response until the second skin test. They are erroneously mistaken for converters.

\section{METHODS}

\section{Study Population}

The ODOC consisted of twelve institutions and one intake center during the study period from July 00-01 (14). The inmate population ranged from $n=166$ at the Oregon Women's Correctional Center to $\mathrm{n}=2,794$ at the Snake River Correctional Institution. Men comprised $95 \%(n=9,746)$ of the inmate population and women
$5 \%(\mathrm{n}=573)$. Three-quarters of the inmates were Caucasian, 11\% Latino, 11\% Black, and 3\% other. One-third of the inmates were between the ages of 18-30, almost half between $31-45$ and one-fifth between 45 and 70 . Due to the demographic preponderance of men, women were excluded from the study.

\section{Confidentiality}

Institutional Review Board (IRB) approval was obtained and inmate names stripped from the records. A unique identifier was used and all the data presented in aggregate form.

\section{Case Selection}

Pharmacy records revealed that 307 men were on anti-TB medications during the study period. This captured $99 \%$ of men with a recent or past positive skin test. Ninety percent of their medical charts containing skin test data were reviewed $(\mathrm{n}=276)$ and the men separated into converters $(n=72)$, reactors $(\mathrm{n}=123)$, and prior positives on medications $(n=81)$. Converters were inmates who tested negative at entry and positive on annual testing. Some of them were positive before the oneyear period $(n=23)$. Reactors were inmates who tested positive at entry, and priors on medications were positive before entry. Ten percent of the records $(n=31)$ were not reviewed and accounted for a potential of 8 missing converters.

\section{Control Selection}

A database manager at the ODOC randomly selected 305 male inmates from all 12 institutions who were never on anti-TB medications. All of these inmates resided in the ODOC from July 00- July 01. Seventy-seven percent $(n=234)$ of the controls were verified through a medical chart review.

\section{Demographic Information}

The demographic variables seen in table 1 were collected from two sources. All the variables were derived from the Correctional Information System (CIS) database except for "drug abuse potential" that came from the mental health computer database. Inmate psychiatric assessments provided the data for that variable.

\section{Data Analysis}

The conversion rate was calculated by dividing the number of converters from the estimated inmateyears during the study period.

For the case-control study, univariate analysis was performed with the use of chi-square for categorical 
Table 1. Demographic characteristics of the cases and verified controls.

\begin{tabular}{|c|c|c|}
\hline Characteristics & $\begin{array}{l}\text { Cases } \\
(\mathrm{n}=72)\end{array}$ & $\begin{array}{l}\text { Verified Controls } \\
(\mathrm{n}=\mathbf{2 3 4})\end{array}$ \\
\hline Age in Years & $33(11)$ & $38(11)$ \\
\hline 20-29 - no. (\%) & $20(27.8)$ & $53(22.7)$ \\
\hline $30-39$ & $23(31.9)$ & $83(35.5)$ \\
\hline $40-49$ & $18(25.0)$ & $62(26.5)$ \\
\hline $50-59$ & $9(12.5)$ & 29 (12.4) \\
\hline $60-69$ & $0(0)$ & $6(2.6)$ \\
\hline $70-79$ & $2(2.8)$ & $1(.43)$ \\
\hline \multicolumn{3}{|l|}{ Race, no. (\%) } \\
\hline Caucasian & $42(58.3)$ & $186(79.5)$ \\
\hline Latino & $22(30.6)$ & $16(6.8$ \\
\hline African-American & $5(6.9)$ & $23(9.8)$ \\
\hline Other & $3(4.2)$ & $9(3.8)$ \\
\hline \multicolumn{3}{|c|}{ Citizenship, no. (\%) } \\
\hline United States & $57(79.2)$ & $229(97.9)$ \\
\hline Mexico & $12(16.7)$ & $4(1.7)$ \\
\hline Other & $3(4.2)$ & $1(0.4)$ \\
\hline \multicolumn{3}{|l|}{ Birthplace, no. (\%) } \\
\hline Oregon & $11(15.3)$ & $77(32.9)$ \\
\hline Other place & $61(84.7)$ & $157(67.1)$ \\
\hline \multicolumn{3}{|l|}{ Birthplace, no. (\%) } \\
\hline United States & $40(55.6)$ & $220(94.0)$ \\
\hline Non-U.S. & $32(44.4)$ & $14(6.0)$ \\
\hline \multicolumn{3}{|c|}{$\begin{array}{l}\text { County of Incarceration, } \\
\text { no. }(\%)\end{array}$} \\
\hline AOC District $1^{*}$ & $1(1.4)$ & $8(3.4)$ \\
\hline AOC District 2* & $6(7.8)$ & $7(3.0)$ \\
\hline AOC District 3* & $3(8.3)$ & $4(1.7)$ \\
\hline AOC District $4^{*}$ & $8(4.2)$ & $27(11.5)$ \\
\hline AOC District $5^{*}$ & $6(8.3)$ & $26(11.1)$ \\
\hline AOC District 6* & $12(16.7)$ & $37(15.8)$ \\
\hline AOC District 7* & $4(5.6)$ & $8(3.4)$ \\
\hline AOC District $8^{*}$ & $26(36.1)$ & $92(39.3)$ \\
\hline Unknown & $6(8.3)$ & $25(10.7)$ \\
\hline \multicolumn{3}{|l|}{ Education, no. (\%) } \\
\hline Un-testable & 0 & $7(3.0)$ \\
\hline Obtained GED* & $20(27.8)$ & $90(38.5)$ \\
\hline No GED* & $52(72.2)$ & $137(58.5)$ \\
\hline \multicolumn{3}{|c|}{$\begin{array}{l}\text { Location of the main } \\
\text { institution of Incarceration, } \\
\text { no. (\%) }\end{array}$} \\
\hline AOC District $1 *$ & $44(61.1)$ & $138(59.0)$ \\
\hline AOC District 2* & 0 & 0 \\
\hline AOC District 3* & 0 & 0 \\
\hline AOC District 4* & $3(4.2)$ & $1(0.4)$ \\
\hline AOC District $5^{*}$ & 0 & 0 \\
\hline AOC District 6* & $17(23.6)$ & $87(37.2)$ \\
\hline AOC District $7^{*}$ & 0 & $2(0.9)$ \\
\hline AOC District $8 *$ & $1(1.4)$ & $3(1.3)$ \\
\hline Unknown & $7(9.7)$ & $3(1.3)$ \\
\hline \multicolumn{3}{|c|}{ Level of Security, no. (\%) } \\
\hline Maximum & $8(11.1)$ & $58(24.8)$ \\
\hline Medium & $6(8.3)$ & $154(65.8)$ \\
\hline Minimum & $51(70.8)$ & $19(8.1)$ \\
\hline Unknown & $7(9.7)$ & $3(1.3)$ \\
\hline \multicolumn{3}{|c|}{$\begin{array}{l}\text { Institutional Density, } \\
\text { no. (\%) }\end{array}$} \\
\hline $\mathrm{High}^{*}$ & $38(52.8)$ & $180(76.9)$ \\
\hline Medium* & $22(30.6)$ & $46(19.7)$ \\
\hline
\end{tabular}

Low* $\quad 5(6.9) \quad 5(2.1)$

Unknown $\quad 7(9.7)$

Drug abuse potential,

no. (\%)

High* $34(47.2) \quad 104(44.4)$

Low* $28(38.9) \quad 123(52.6)$

Unknown $\quad 10(13.9) \quad 7(3.0)$

Prior number of

incarcerations-

mean (SD)

$92(2.25)$

$1.20(2.28)$

Number of visits

in one year- mean (SD) $10(18) \quad 20(37)$

Number of visitors

in one year- mean (SD) $21(36)$

Number of PPD

skin tests- mean (SD) $\quad 2.7(1.2) \quad 4.4(2.3)$

Duration of incarceration

prior to conversion or

July 2001 in days-

$609(539)$

$1278(1071)$

Number of institutions

inhabited- mean (SD)

$2.5(1.1)$

$2.4(1.5)$

Number of relocations

to other prisons-

mean (SD)

$1.6(1.1)$

$1.7(1.9)$

variables and the student t-test for continuous variables. Multivariate analysis was performed with logistic regression. Pearson's correlation was used to choose variables for the logistic model. Variables that were heavily correlated were grouped together and only the most significant ones entered into the main effects model. The best models were presented with Odd Ratios (OR) and 95\% confidence intervals.

\section{RESULTS}

\section{Demographic Characteristics of Cases and} Verified Controls

Thirty percent of the cases $(n=22)$ were Latino, while only seven percent $(n=16)$ of the controls were of the Latin race. More of the cases had Mexican citizenship ( $n=12$ or $17 \%$ versus $n=4$ or $1.7 \%$ for the controls) and were born in a non-U.S. country ( $n$ $=32$ or $44 \%$ vs. $\mathrm{n}=14$ or $6 \%$ ). The cases were more likely to live in minimum security prisons $(\mathrm{n}=51$ or $71 \%$ vs. $\mathrm{n}=19$ or $8 \%$ ), and less likely to live in high density prisons $(\mathrm{n}=38$ or $53 \%$ vs. $\mathrm{n}=180$ or $77 \%)$. They had fewer PPD skin tests (2.7 vs. 4.4) but lived in more institutions ( 2.5 vs. 2.4$)$. The other variables are presented in Table 1. 


\section{Conversion Rate}

The conversion rate was 5.0 per 1,000 person-years. Forty-nine of the seventy-two converters were positive from July 00 - July 01 . The estimated person-years were 9,746 inmate-years.

\section{Validity of the Controls}

With all the demographic information available on the controls, the verified control sample $(n=234)$ was compared to the entire control sample $(n=305)$. There was no statistically significant difference between the two samples (chi-square, $\mathrm{p}<.36$ to .99 ; t-test, $\mathrm{p}<.35$ to $.73)$.

\section{Univariate Analysis}

The cases were more likely to be non-white $(\mathrm{p}<.001)$, to have foreign citizenship $(\mathrm{p}<.001)$, and to be born outside of Oregon $(\mathrm{p}<.001)$ or in a foreign country $(\mathrm{p}$ $<.004)$. They tended to live in different districts $(\mathrm{p}<$ $.048)$, different security prisons $(\mathrm{p}<.001)$, and different density institutions $(\mathrm{p}<.003)$ than the controls. They received fewer visits $(\mathrm{p}<.02)$, fewer PPD skin tests $(\mathrm{p}$ $<.001)$, and had a shorter duration of stay $(\mathrm{p}<.001)$.

\section{Correlation Analysis}

Race, citizenship, and birthplace were positively correlated ( $\mathrm{p}<.001-.005)$. Many Latinos were Mexican citizens born in Mexico. The level of security was positively correlated with the institutional density $(\mathrm{p}<$ $.001)$. Self-evidently correlated were the number of visitors and visits $(p<.001)$. The number of PPD skin tests and duration of residence were positively correlated as well $(\mathrm{p}<.001)$. Inmates with longer residences had more annual PPD skin tests. However, the duration of residence $\&$ number of PPD skin tests were negatively correlated with the number of institutions lived in $(p<.001)$. It appears that inmates who enter the prison system move around multiples times initially before settling down in one location.

\section{Multivariate Analysis}

Sixteen models were tested based on variations of the correlated variables. If race was entered, birthplace and citizenship were left out. If security was used, then density was removed. The three models containing the greatest number of significant risk factors are displayed in Table 2.

Based on the three models, the cases were 6 times more likely to be Latino, 10 times more likely to be born outside the U.S., and 13 times more likely to have Mexican citizenship. They were 71-77 times less likely to live in medium vs. minimum security prisons and 1923 times less likely to live in maximum vs. minimum security prisons. The cases had 1.4-1.5 times fewer PPD skin tests and lived in 1.5-1.7 times fewer prisons. On average, the cases lived in more institutions $(n=2.51$ vs. 2.43 ), but a greater proportion of cases (88\% vs. $78 \%$ ) lived in 3 or fewer institutions, accounting for the trend.

Based on the other thirteen models where only three variables were significant, the cases were 2-3 times more likely to be born outside of Oregon, 5-7 times less likely to live in medium vs. low density prisons, and 611 times less likely to live in high vs. low density prisons.

Table 2. Best logistic regression models

\begin{tabular}{|c|c|c|c|}
\hline Model One & OR & $95 \% C I$ & P-Value \\
\hline \multicolumn{4}{|l|}{ Birthplace } \\
\hline (non-U.S. vs. U.S.) & 9.87 & $3.06-31.8$ & .001 \\
\hline \multicolumn{4}{|l|}{ Security } \\
\hline (Max vs. Min) & .052 & $.017-.158$ & .001 \\
\hline \# PPD skin tests & .696 & $.529-.915$ & .009 \\
\hline Number of institutions & .639 & $.443-.922$ & .017 \\
\hline Model Two & OR & $95 \% \mathrm{CI}$ & P-Value \\
\hline \multicolumn{4}{|l|}{ Race } \\
\hline (Latino vs. White) & 5.98 & $1.70-21.1$ & .005 \\
\hline (Black vs. White) & .748 & $.157-3.56$ & .716 \\
\hline (Other vs. White) & 1.74 & $.172-17.5$ & .640 \\
\hline \multicolumn{4}{|l|}{ Security } \\
\hline (Med vs. Min) & .013 & $.004-.040$ & .001 \\
\hline (Max vs. Min) & .049 & $.017-.142$ & .001 \\
\hline \# PPD skin tests & .649 & $.492-.856$ & .002 \\
\hline Number of institutions & .585 & $.401-.851$ & .005 \\
\hline Model Three & OR & $95 \%$ CI & P-Value \\
\hline \multicolumn{4}{|l|}{ Citizenship } \\
\hline (Mexican vs. U.S.) & 13.0 & $1.77-95.1$ & .012 \\
\hline (Other vs. U.S.) & 7.28 & $.327-162$ & .210 \\
\hline \multicolumn{4}{|l|}{ Security } \\
\hline (Med vs. Min) & .014 & $.005-.042$ & .001 \\
\hline (Max vs. Min) & 7.28 & $.327-162$ & .001 \\
\hline \# PPD skin tests & .650 & $.490-.861$ & .003 \\
\hline Number of institutions & .587 & $.406-.850$ & .005 \\
\hline
\end{tabular}

\section{DISCUSSION}

The conversion rate in the ODOC was very low compared to other prisons during the epidemic from 1985-1992. Prisons with intramural conversion had average rates between 39 and 67 per 1,000 person-years $(11,15-16)$. In the Oregon prisons, the known risk factors for intramural conversion were not seen. The 
converters lived in prisons with fewer inmates and stayed for shorter durations of time. Their hypothetical exposure to TB was lower compared to the controls in the Oregon prisons.

There are only a few possibilities that can explain the initial negative skin test seen in the 49 converters from July $00-01: 1$ ) anergy, 2) incubating disease at admission, 3) intramural transmission, and 4) the booster phenomenon. Anergy is a state of depressed immune response to multiple antigens, while the booster phenomenon is a transient decreased immune response to the antigen in the PPD skin test. The anergic individual is immuno-suppressed, but the 'booster' is often immuno-competent and simply needs the first skin test to 'boost' the immune response to the PPD antigen.

In this study, anergy was not a possible explanation since the converters tested positive on subsequent skin tests. Furthermore, over $60 \%$ of the conversions occurred on the second skin test, suggesting the boosting phenomenon. Second, incubating disease at admission is possible, but unlikely to differentially affect Latino men. This would affect all inmates equally. Third, there has only been one active case of TB diagnosed in the Oregon prisons from 2000-2001. It is possible, but very unlikely that this single inmate or a few undiagnosed inmates preferentially infected the Latino males who lived in different institutions. Therefore, intramural conversion seems less likely than the last alternative- the booster phenomenon. The high percentage of conversions on the second skin test and lack of another plausible explanation argue in favor of the booster phenomenon.

The risk factors for boosting have not been studied in the prison population according to the author's literature searches. Research on health care workers, school children, and young adults showed older age, previous BCG vaccination, and sensitivity to atypical Mycobacterium to be risk factors (17-23). Older age decreases the immune response to the skin test antigen, and previous vaccination or sensitivity to atypical Mycobacterium elicits a weaker response respectively. The converters were young to middle-age and only $11 \%$ were fifty or older. Regarding the BCG, Mexico does not give these vaccinations. Previous sensitivity to atypical Mycobacterium is a possible explanation.

In the Maryland prisons, the rate of boosting was $1 \%$ (24). The health officials there did not think it was costeffective to initiate two-step skin testing. The question is whether to implement two-step skin testing in the Oregon Department of Corrections. By testing inmates twice, the booster phenomenon can be evaluated. Two negative tests suggest the absence of infection, while a negative followed by a positive test suggests the booster phenomenon. A boosting study would reveal whether Latino men are specifically at risk for conversion. It would give a definitive answer to true vs. false conversion.

At the maximum, the rate of boosting was .46 per 100 inmates, or less than half a percent. It would take 200 extra skin tests to discover one booster in the Oregon prisons. More than one thousand inmates would have to be tested to perform an adequate study. It does not appear cost-effective to test the general inmate population, but testing a subset of Latino men would be both practical and feasible.

Regarding validity, misclassification from the eight potential converters could not change the results. Eight additional 'dummy' inmates were coded in the opposite direction of the results. The Odd Ratios were reduced but remained significant. In addition, ten percent of the data set was double-checked. If $>5 \%$ of the data reviewed was inaccurate, the entire variable was recoded again.

Overall, the case-control study was efficient and unique. It used existing data to determine the risk factors for conversion. Only three other prison systems have done this in the U.S.. (11, 15-16) More importantly, a computerized TB registry and the aforementioned boosting study may soon be implemented. Conversion rates can be followed yearly without the need to perform site visits, and a subset of men may be skin-tested twice in the future.

\section{ACKNOWLEDGEMENTS:}

The author thanks Dr. Gary Sexton, PhD, for statistical assistance, Dr. Eldon Edmundson, $\mathrm{PhD}$ for editing the manuscript, and Dr. Jay Kravitz, MD, MPH for discussions on tuberculosis. This research was supported in part by PHS grant 5 M01 RR00334.

\section{REFERENCES}

1. Centers for Disease Control. World TB Day 2003- Global Fact Sheet. The Impact of Tuberculosis Worldwide.

2. CDC Core Curriculum on Tuberculosis. What the Clinician Should Know. 4th edition, 2000.

3. Braun MM, Truman, BI, Maguire B. Increasing incidence of tuberculosis in a prison inmate population: association with HIV infection. Journal of American Medical Association, 261: 393-7, 1989.

4. Tuberculosis Control Program. Annual Report, 1994. Trenton, NJ: New Jersey Department of Health, 1995.

5. CDC. Probable transmission of multi-drug-resistant tuberculosis in a correctional facility - California. Morbidity \& Mortality Weekly Report, 42: 48-51, 1993.

6. Prevention and Control of Tuberculosis in Correctional Institutions: Recommendations of the Advisory Committee for the Elimination of Tuberculosis. Morbidity \& Mortality Weekly Report, 38(18): 313-325, 1989.

7. CDC: Controlling TB in Correctional Facilities. Atlanta, GA: U.S. Department of Health and Human Services, 1995. 
8. CDC. Reported Tuberculosis in the United States, 2002. Atlanta, GA: U.S. Department of Health and Human Services, 2003.

9. Oregon 2002 Community TB Profile. Oregon TB Control. HIV/STD/TB Program. Dept. of Health Services, 2003.

10. Ending Neglect: The Elimination of Tuberculosis in the United States. Lawrence Geiter, ed. Committee for the Elimination of Tuberculosis in the United States. Division of Health Promotion and Disease Prevention. 292, pages, 2000.

11. MacIntyre CR, Kendig N, Kummer L, Birago, S, Graham N. Impact of Tuberculosis Control Measures and Crowding on the Incidence of Tuberculous Infection in Maryland Prisons. Clinical Infectious Diseases, 24: 1060-70, 1997.

12. March F, Coll P, Guerrero RA, Busquets E, Cayla JA, Prats G. Predictors of tuberculosis transmission in prisons: an analysis using conventional and molecular methods. Acquired Immunedeficiency Syndrome, 14: 525-535, 2000.
13. Bellin EY, Fletcher DD, Safyer SM. Association of Tuberculosis Infection With Increased Time in or Admission to the New York City Jail System. Journal of American Medical Association, Vol. 269, No. 17, 1993.

14. Oregon Department of Corrections Statistics, 2000-2001.

15. Spencer SS, Morton AR. Tuberculosis Surveillance in a State Prison. American Journal of Public Health, 79(4): 5079, 1989.

16. Johnsen, C. Tuberculosis contact investigation: Two years experience in New York City correctional facilities. American Journal of Infection Control, 21: 1-4, 1993.

17. Hoft DF, Tennant JM. Persistence and Boosting of Bacille Calmette-Guerin-Induced Delayed-Type Hypersensitivity. Annals of Internal Medicine, 131: 32-36, 1999.

18. Menzies R, Vissandjee B, Rocher I, Germain, Y. Annals of Internal Medicine, 120: 190-98, 1994.

Robert Hung is a fourth year medical student at Oregon Health Sciences University. He holds a B.S. in Biology from Stanford University, California, U.S.A. He will begin a medicine and psychiatry residency at RUSH in Chicago, IL in July 2003. He hopes to become a correctional physician in the near future. 